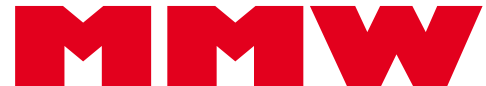

Fortschritte der Medizin

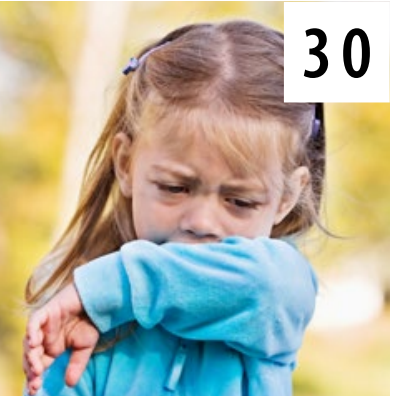

COVID-19 bei Kindern

Die Vermutung, dass Kinder und Jugendliche sich weniger häufig mit SARS-CoV-2 infizieren, ist wohl nicht mehr haltbar.
Gewalt gegen Ärzte

Lesen Sie im Report, wie Sie sich gegen pöbelnde Patienten oder übergriffige Kollegen wehren können.

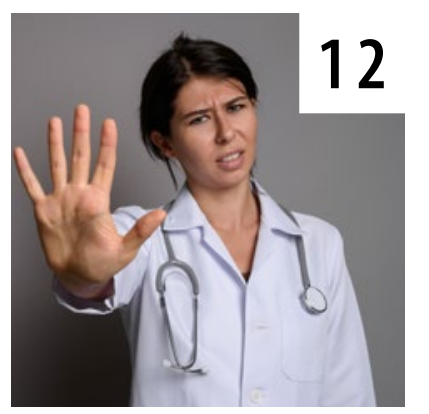

Videosprechstunde

Es gibt immer neue

Möglichkeiten - und immer mehr Honorar. Der aktuelle Überblick.

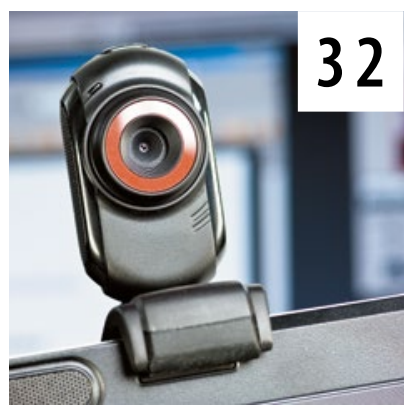

\section{Aktuelle Medizin}

Blickdiagnose

09 Verursacht ein Tumor die epigastrischen Schmerzen?

10 Magazin

\section{Report}

12 Gewalt gegen Ärzte: Mit aggressiven Patienten und Kollegen fertig werden

Impfsprechstunde

18 Wichtige Indikationsimpfungen: Der zuverlässigste Schutz vor Infektionen - gerade auch für Diabetiker

Aktuelle Berichte

20 Aus Angst vor Corona nicht zum Arzt?

22 Antikoagulation bei schwerer Niereninsuffizienz

\section{Kritisch gelesen}

Aktuelle Studien

26 Feinstaubbelastung erhöht Depressionsrisiko

28 Nierenschwache Senioren vertragen Kontrastmittel

30 COVID-19: Rätsel um den Verlauf bei Kindern

Von H. J. Heppner, J. Zeeh, D. Reinhardt

Internationale Fallberichte

27 Hypertonieschäden am Auge

28 So können weiße Europäer schwarz werden Von H. Holzgreve

\section{Aus der Praxis}

Von Hausarzt zu Hausarzt

32 Und plötzlich geht immer mehr per Video

33 COVID-19: Ärzte können auf Finanzhilfe hoffen

Von G. W. Zimmermann, Hofheim

Hotline

34 Stellungnahme für Betreuung abrechnen

Von H. Walbert, Würzburg

35 Geschichten aus der Praxis 


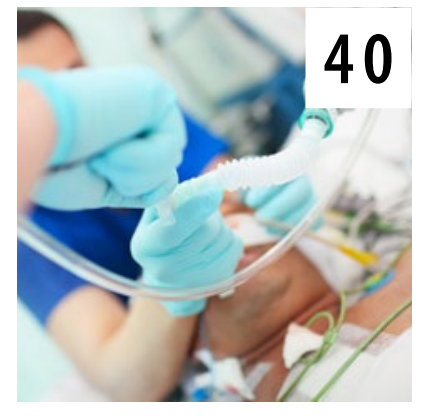

COVID-19 im Alter:

Beatmen oder palliativ

behandeln?

Der Beitrag nennt Kriterien, wie medizinisch, ethisch und rechtlich vertretbar entschieden werden kann.

Corona-Ambulanzen

Hier erfahren Sie, wie eine Corona-Ambulanz aufgebaut und organisiert ist.

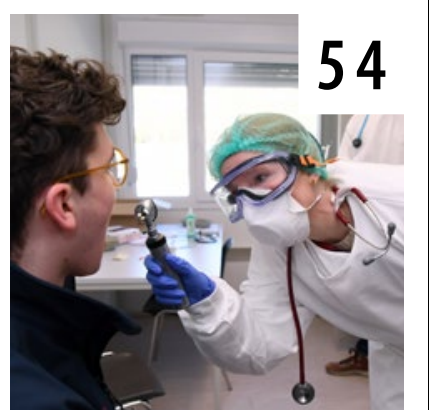

Redaktion:

$089 / 203043-1352$

redaktion.mmw@springer.com

Abonnentenservice: 06221/345-4304

www.springermedizin.de/mmw

MMW-Fortschritte der Medizin erscheint in Fortsetzung von

MMW MÜNCHENER MEDIZINISCHE WOCHENSCHRIFT,

begründet 1853 in München, und

FORTSCHRITTE DER MEDIZIN, begründet 1882 durch

Robert Koch und andere Fachgelehrte in Berlin

\section{Fortbildung}

Schwerpunkt SARS-CoV-2-Pandemie

36

COVID-19 im Alter

Übersicht von M. Gosch, Nürnberg, et al.

40 Beatmung geriatrischen Patienten - ein ethisches

Dilemma?

Übersicht J. Zeeh, K. Memm, H.-J. Heppner, A. Kwetkat

45 Advance Care Planning

Übersicht von B. Feddersen, München, et al.

48 Priorisierung von Pflegeheimbewohnern aus notfallmedizinischer Sicht

Übersicht von G. Michels, Eschweiler, et al.

51 Corona-Pandemie: Herausforderung für die pflegerische Versorgung in der stationären Altenhilfe

Übersicht von M. Halek, Witten, et al.

54 Behandlung von Patienten in Corona-Ambulanzen

Übersicht von M. Bleckwenn, Leipzig

57 Ernährung in Zeiten der Corona-Pandemie

Übersicht von H. Hauner, München

60 COVID-19 in einer stationären Pflegeeinrichtung

Kasuistik von U. Grabenhorst, Viersen, et al.
64 COVID-19-Cluster-Studie an einem Lehrkrankenhaus Originalie von A. von Freyburg, Dachau, et al.

Weitere Fortbildungsbeiträge

67 RAS-Inhibition in Zeiten der COVID-19-Pandemie

Seminar von F. Limbourg, Hannover

71 Schlafapnoe

Seminar von M. Barczok, Ulm

\section{Pharmaforum}

75 Impressum

Mehr Service für Sie:

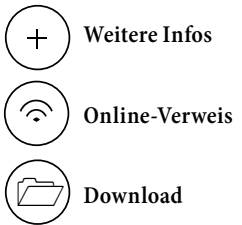

